\title{
Correction to: Weak nil clean ideals
}

\author{
Ajay Sharma ${ }^{1} \cdot$ Dhiren Kumar Basnet $^{1}$
}

Published online: 24 August 2019

(c) Springer-Verlag Italia S.r.l., part of Springer Nature 2019

\section{Correction to: Rendiconti del Circolo Matematico di Palermo Series 2 https://doi.org/10.1007/s12215-019-00426-z}

The authors used the notion of Nil clean rings, which was introduced by P. V. Danchev and W. Wm. McGovern in their paper "Danchev P.V., McGovern W.Wm., Commutative weakly nil clean unital rings, J. Algebra 425 (2015), 410-422.”. But unfortunately, it was not cited in the paper. So the reference no. 2 of the paper should be replaced by the paper of P. V. Danchev and W. Wm. McGovern. The authors regret for the mistake and would like to thank Prof. Danchev for pointing it out.

\section{References}

1. Danchev, P.V., McGovern, WWm: Commutative weakly nil clean unital rings. J. Algebra 425, 410422 (2015)

2. Breaz, S., Danchev, P., Zhou, Y.: Rings in which every element is either a sum or a difference of a nilpotent and an idempotent. J. Algebra Appl. 15 (2016)

Publisher's Note Springer Nature remains neutral with regard to jurisdictional claims in published maps and institutional affiliations.

The original article can be found online at https://doi.org/10.1007/s12215-019-00426-z.

Dhiren Kumar Basnet

dbasnet@tezu.ernet.in

Ajay Sharma

ajay123@tezu.ernet.in

1 Department of Mathematical Sciences, Tezpur University, Sonitpur, Napaam, Assam 784028, India 